density $(p<0.02)$ and haemorrhages $(p<0.01)$ in the initial NVC, as well as the presence of ramifications in the control NVC $(p<0,05)$.

It was observed that patients with normal capillary organization presented better value of FVC $(p<0.01)$, TLC $(p<0.01)$, and lower FEV1/FVC ratio $(p<0.02)$, the latter finding also found in control NVC $(p<0.03)$. As additional data, we found that patients with anti-Ku+ presented better values of FVC $(p<0.04)$ and TLC $(p<0.05)$, but although they all had normal capillary organization, the association of this antibody with NVC was not statistically significant. We also did not find a statistically significant relationship between the alterations in NVC and the presence of Raynaud's phenomenon, the other clinical variables, cancer history and the presence of overlap syndromes.

\begin{tabular}{|c|c|}
\hline Table 1 & PATIENTS \\
\hline $\mathrm{N}$ & 20 \\
\hline Age at diagnosis (years) & $55 \pm 11,7$ \\
\hline Time of evolution (years) & $2,9 \pm 1,8$ \\
\hline Active smoking & $5(25 \%)$ \\
\hline Muscle weakness & $7(3556)$ \\
\hline CK elevation at diagnosis & $8(4096)$ \\
\hline EMG compatible with IM & $5 / 8(62.5 \%)$ \\
\hline Muscle biopgr compatible with IM & $7 / 8(87,596)$ \\
\hline Skin findings & $6(3056)$ \\
\hline Cardiac disease & $1(5 \%)$ \\
\hline Disphagia & $5(25 \%)$ \\
\hline Lung disease & $7(35 \%)$ \\
\hline Concomitant cancer & $1(5 \%)$ \\
\hline Raynaud's phenomenon present & $11(55 \% 6)$ \\
\hline Overlap: & $5(25 \%)$ \\
\hline - With Systemic Sclerosis & $\begin{array}{l}4 / 5(80 \% 6) \\
1 / 5(206)\end{array}$ \\
\hline -With Sjögren Syndrome & \\
\hline Anti-Mi2 & $4(2056)$ \\
\hline Anti-Ku & $5(25 \% 6)$ \\
\hline Anti-Jo1 & $2(1096)$ \\
\hline Anti-PL7 & $2(1056)$ \\
\hline Anti-Ro52 & $5(25 \% 6)$ \\
\hline Other Ab (SRP, PL12, PM/SCL, OJ, MDAS, TIF1G) & $6(30 \%)$ \\
\hline
\end{tabular}

Conclusions: Patients with capillary disorganization in NVC showed worse values of FVC, TLC and FEV1/FVC. We found a statistically significant association between esophageal disease and haemorrhages, loss of capillary density and ramifications. Prospective studies with larger sample sizes are required to define the usefulness of NVC in the diagnosis, prognosis and follow-up of these patients. Disclosure of Interest: None declared

DOI: 10.1136/annrheumdis-2017-eular.6730

\section{SAT0380 CLASSIFICATION, CATEGORISATION AND ESSENTIAL ITEMS FOR DIGITAL ULCER (DU) EVALUATION IN SYSTEMIC SCLEROSIS (SSC): A DESSCIPHER/EUSTAR SURVEY}

J. Blagojevic ${ }^{1,2,3}$, L. Cometi ${ }^{1}$, G. Abignano ${ }^{3,4}$, S. Guiducci ${ }^{1}$

S. Bellando-Randone ${ }^{1}$, D. Huscher ${ }^{5}$, J. Avouac ${ }^{6}$, L. Czirják ${ }^{7}$, C. Denton ${ }^{8}$

O. Distler ${ }^{9}$, M. Frerix ${ }^{10}$, V.K. Jaeger ${ }^{11}$, V. Lóránd ${ }^{7}$, B. Maurer ${ }^{9}$, S. Nihtyanova ${ }^{8}$,

G. Riemekasten ${ }^{12}$, E. Siegert ${ }^{13}$, G. Valentini ${ }^{14}$, S. Vettori ${ }^{14}$, U. Walker ${ }^{15}$,

U. Müller-Ladner ${ }^{10}$, Y. Allanore ${ }^{16}, F$. Del Galdo ${ }^{3,4}$, M. Matucci-Cerinic ${ }^{1}$ on behalf of DeSScipher Consortium and contributing EUSTAR centres. ${ }^{1}$ Department of Experimental and Clinical Medicine, University of Florence, Florence, Italy;

${ }^{2}$ Leeds Institute of Rheumatic and Musculoskeletal Medicine, University of Leeds; ${ }^{3}$ NIHR Leeds Musculoskeletal Biomedical Research Unit, Leeds Teaching Hospitals NHS Trust; ${ }^{4}$ Leeds Institute of Rheumatic and Musculoskeletal Medicine, University of Leeds, Leeds, United Kingdom; ${ }^{5}$ Department of Rheumatology and Immunology, Charité University Hospital and German Rheumatism Research Centre, Berlin, Germany; ${ }^{6}$ Department of Rheumatology, University of Paris Descartes, Paris, France; ${ }^{7}$ Department of Rheumatology and Immunology, University of Pécs, Pécs, Hungary; ${ }^{8}$ Department of Rheumatology, University College London, Royal Free Hospital, London, United Kingdom; ${ }^{9}$ Department of Rheumatology, University Hospital Zurich, Zurich, Switzerland; ${ }^{10}$ Department of Rheumatology and Clinical Immunology, Justus-Liebig University Giessen, Kerckhoff Clinic Bad Nauheim, Giessen/Bad Nauheim, Germany; ${ }^{11}$ Department of Rheumatology, University of Basel, Basel, Switzerland; ${ }^{12}$ University Schleswig-Holstein Lübeck, Lübeck; ${ }^{13}$ Department of Rheumatology and Immunology, Charité University Hospital, Berlin, Germany; ${ }^{14}$ Department of Rheumatology, Second University of Naples, Naples, Italy; ${ }^{15}$ Department of Rheumatology, University of Basel, Basel, Switzerland;

${ }^{16}$ Department of Rheumatology, University of Paris Descartes, Paris, France

Background: A consensus on DU definition in SSc has been recently reached (1),while for their evaluation,classification and categorisation it is still missing. Objectives: To identify in SSc a set of essential items for DU evaluation, to assess if the existing DU classification (2) was useful and feasible in clinical practice, and to investigate if the DU categorisation (3) was preferred to the simple division of DU in recurrent and not recurrent.

Methods: The DU Desscipher items that reached $>60 \%$ of completion rate were administered to EUSTAR centres via online survey. These items were: DU distal to the proximal interphalangeal joints, recurrent DU, DU history, infection, gangrene, amputation, total number of DU, number of new DU, number of healed DU, number of DU defined as loss of tissue, number of DU due to calcinosis and number of DU due to digital pitting scars (DPS).Questions about feasibility and usefulness of the existing DU classification (DU due to DPS, to loss of tissue, derived from calcinosis and gangrene) (2) and newly proposed DU categorisation (episodic, recurrent and chronic) (3) were also administered.

Results: All Desscipher and 82/194 EUSTAR centres (42.3\%) completed the questionnaire.Out of 27 items selected for the Desscipher study, those scored by $>70 \%$ of participants as essential and feasible for DU evaluation in clinical practice were only the following: number of DU defined as a loss of tissue (level of agreement $91.1 \%)$, recurrent DU (84\%) and number of new DU (74.4\%).For $64.6 \%$ of the centres,the classification of DU was considered useful and feasible in clinical practice.Moreover, $80.3 \%$ of the centres preferred the categorization of DU in episodic,recurrent and chronic.

Conclusions: For clinical practice, EUSTAR centres identified only three essential items for DU evaluation and considered useful and feasible the proposed classification and categorisation of DU.The set of items needs to be further validated by Delphi voting in order to implement its use in clinical practice while further implementation of DU classification and categorisation is warranted.

\section{References:}

[1] Suliman Y et al.Preliminary musculoskeletal ultrasound (MSUS) ulcer definition does not correlate with visual observation in systemic sclerosis (SSc) patients. J. Scleroderma Relat. Disord.2017(in press).

[2] Amanzi L et al.Digital ulcers in scleroderma: staging, characteristics and subsetting through observation of 1614 digital lesions. Rheumatology (Oxford) 2010:49:1374-82.

[3] Matucci-Cerinic $\mathrm{M}$ et al.Elucidating the burden of recurrent and chronic digital ulcers in systemic sclerosis: long-term results from the DUO Registry. Ann Rheum Dis. 2016;75:1770-6.

Disclosure of Interest: None declared

DOI: 10.1136/annrheumdis-2017-eular.6815

\section{SAT0381 CORRELATION BETWEEN THREE DIFFERENT METHODS TO ASSESS DERMAL THICKNESS IN SYSTEMIC SCLEROSIS PATIENTS WITH DIFFERENT PATTERNS OF NAILFOLD MICROANGIOPATHY}

B. Ruaro, A. Sulli, E. Alessandri, S. Paolino, M. Ghio, A.C. Trombetta, C. Pizzorni, V. Tomatis, M. Cutolo. Research Laboratory and Academic Division of Clinical Rheumatology, Department of Internal Medicine, University of Genova, IRCCS AOU San Martino, Genoa, Italy

Background: Systemic sclerosis (SSc) is characterized by the increase of dermal thickness (DT) (1). The modified Rodnan skin score (mRss) is the validated method to evaluate the severity of skin impairment $(2,3)$. Several studies have reported the capability of high frequency skin ultrasound (US) to reflect the overall severity of the skin damage in SSc patients (4-5). The plicometer skin test (PST) is another method to evaluated cutaneous involvement in SSc patients (6).

Objectives: The aim of this study was to identify possible correlations between US, mRss and PST to evaluate DT in SSc patients with different patterns of nailfold microangiopathy.

Methods: Sixty-three SSc patients (mean age $64 \pm 11$ SD years, mean disease duration $7 \pm 6$ years, $43 \mathrm{IcSSc}$ and $20 \mathrm{dcSSc}$ ) and 63 sex and age matched healthy subjects were enrolled after written informed consent. All subjects were assessed by mRss, US and PST to evaluate the DT in the seventeen skin areas of the body usually evaluated by mRss (zygoma, fingers, hands, dorsum of hands, forearms, arms, chest, abdomen, thighs, legs, feet) (1-6). Nailfold videocapillaroscopy (NVC) was used to assess the proper pattern of microangiopathy and to calculate the microangiopathy evolution score (MES) (7-8). Statistical evaluation was performed by non-parametric tests.

Results: As expected, the group of SSc patients had a statistically significant higher DT, as evaluated by the three methods, at level of all areas when compared to the control group $(\mathrm{p}=0.0001)$. All methods demonstrated a progressively higher DT in patients with "Early", vs. "Active" and vs "Late" pattern of nailfold microangiopathy $(p<0.005)$, and a positive correlation was observed with MES $(r=0.71 p<0.001)$. A positive correlation was observed in SSc patients between the three method to evaluate DT (PST vs mRss $r=0.98, p<0.0001$; PST vs US $r=0.53, p<0.0001$; US vs mRss $r=0.53, p<0.0001$ ).

Conclusions: This study demonstrates a relationship between different methods to assess DT (US, mRss and PST) in SSc patients and a relationship between skin damage and microangiopathy impairment.

References:

[1] Moore TL, et al. Rheumatology 2003;42:1559-63.

[2] Sulli A, et al. Ann Rheum Dis. 2014;69:1140-3.

[3] Kaldas M, et al. Rheumatology 2009:48:1143-6.

[4] Hesselstrand R, et al. Rheumatology 2008;47:84-7.

[5] Kaloudi O, et al. Ann Rheum Dis. 2010:69:1140-3.

[6] Parodi MN, et al. Br JRheumatol. 1997;36:244-50.

[7] Sulli A, et al. Ann Rheum Dis 2008;67:885-7.

[8] Cutolo et al. J Rheumatol 2000; 27;155.60.

Disclosure of Interest: None declared

DOI: 10.1136/annrheumdis-2017-eular.273 